\title{
Idiopathic pulmonary fibrosis in patients with early-stage non-small-cell lung cancer after surgical resection
}

\author{
Hribernik Nezka ${ }^{1}$, Pozek Igor ${ }^{2}$, Kern Izidor $^{2}$ \\ ${ }^{1}$ Institute of Oncology Ljubljana, Ljubljana, Slovenia \\ 2 University Clinic of Respiratory and Allergic Diseases Golnik, Golnik, Slovenia
}

Radiol Oncol 2019; 53(3): 357-361.

Received 22 January 2019

Accepted 26 June 2019

Correspondence to: Izidor Kern, M.D., Department of Pathology, University Clinic of Respiratory and Allergic Diseases Golnik, Golnik 36, 4204 Golnik, Slovenia. Phone: +386 42569 415; E-mail: izidor.kern@klinika-golnik.si

Disclosure: No potential conflicts of interest were disclosed.

Background. The outcomes of patients with both lung cancer and idiopathic pulmonary fibrosis (IPF) are unfavorable. Therapeutic interventions for lung cancer such as surgery can cause acute exacerbation of IPF (aelPF). This study aimed to assess the frequency of IPF in a group of patients with early-stage non-small-cell lung cancer (NSCLC) and to report clinical characteristics and outcomes of this cohort of patients.

Patients and methods. This observational cohort retrospective study analyzed 641 pathological records of patients after surgical resection of early-stage non-small-cell lung cancer (NSCLC) at University Clinic Golnik from May 2010 to April 2017. Pathological records of NSCLC with coexisting IPF were reviewed. CT scans and biopsy specimens for this group of patients were analyzed by a thoracic radiologist and pathologist, independently. We searched radiological and pathological features of usual interstitial pneumonia (UIP) pattern in this group of patients. We report the clinical characteristics and outcome of this cohort of patients.

Results. Out of 641 patients with early-stage NSCLC, only $13(2.0 \%)$ had histologically and radiologically proven coexisting UIP/IPF. Squamous cell carcinoma was the most common type of lung cancer (7/13 patients). The majority of tumors were small size (all being PT1 or PT2), stage I-II (11/13 patients), located in the lower lung lobes (11/13 patients). Almost all patients were current or ex-smokers (11/13 patients). There were two pathologically confirmed fatal cases (15.4\%) due to aelPF in the first two months after radical treatment, one after adjuvant radiotherapy and the other after surgery. Out of 13 patients, one patient had a lung cancer relapse.

Conclusions. Frequency of UIP/IPF in surgically treated early stage NSCLC is rather low. Our observational study shows that radical treatment of lung cancer can cause aelPF with dismal outcome in this group of patients. The standard of care in these mostly elderly patients still remains unresolved.

Key words: early-stage non-small-cell lung cancer; idiopathic pulmonary fibrosis; acute exacerbation; surgery; radiotherapy

\section{Introduction}

In Slovenia, lung cancer is still the leading cause of cancer mortality in men and the third cause of cancer mortality in women. ${ }^{1}$ Non-small-cell lung cancer (NSCLC) represents about $85 \%$ of all lung cancers. ${ }^{2}$ For $20-25 \%$ of all lung cancer patients, who are diagnosed at an early stage (stage IA-IIIA), surgery remains the best chance of cure. ${ }^{3}$ Patients at very high risk for surgery-related complications can be treated with curative radiotherapy (RT), either hypofractioned high-dose RT or stereotactic body radiation therapy (SBRT) in stage I NSCLC. ${ }^{3}$ Reported local control after SBRT reaches around $80 \%-90 \%$ at 5 years. ${ }^{4,5}$

Idiopathic pulmonary fibrosis (IPF) is a devastating lung disease that affects mostly smokers. It is a specific form of a progressive fibrotic interstitial 
lung disease of unknown cause, occurring primarily in older adults. It shows histopathologic and radiologic pattern of usual interstitial pneumonia (UIP). ${ }^{6}$ Long-term survival of IPF patients still remains poor with median survival rate from 2 to 3 years from time of diagnosis. ${ }^{7}$

Data suggest an increased risk of lung cancer among patients with IPF. ${ }^{8}$ Treating patients with early-stage lung cancer and coexisting IPF can be

TABLE 1. Baseline clinical, radiological and pathological characteristics of patients with early-stage non-small cell lung cancer and idiopathic interstitial pneumonia

\begin{tabular}{|c|c|c|}
\hline Characteristics & & $N=13(\%)$ \\
\hline \multirow{2}{*}{ Gender } & Male & $11(84.6)$ \\
\hline & Female & $2(15.4)$ \\
\hline \multicolumn{2}{|l|}{ Age, years } & $73.3 \pm 4$ \\
\hline \multirow{3}{*}{ Smoking status } & Current smoker & $3(23.1)$ \\
\hline & Former-smoker & $8(61.5)$ \\
\hline & Never-smoker & $2(15.4)$ \\
\hline \multicolumn{2}{|l|}{$\mathrm{FEV}_{1} / \mathrm{FVC}$ before operation } & $0.71 \pm 0.08$ \\
\hline \multirow{2}{*}{$\mathrm{FEV}_{1}$ before operation } & $\mathrm{ml}$ & $2769 \pm 690$ \\
\hline & $\%$ & $101.6 \pm 20$ \\
\hline \multirow{2}{*}{ FVC before operation } & $\mathrm{ml}$ & $3910 \pm 818$ \\
\hline & $\%$ & $105 \pm 19$ \\
\hline DLCO before operation & $\%$ & $74.2 \pm 21$ \\
\hline \multirow{3}{*}{ Histological subtype } & Squamous cell carcinoma & $7(53.8)$ \\
\hline & Adenocarcinoma & $5(38.5)$ \\
\hline & Adenosquamous cell carcinoma & $1(7.7)$ \\
\hline \multirow{8}{*}{$\begin{array}{l}\text { Pathologic TNM stage } \\
\text { ( } 8^{\text {th }} \text { edition) }\end{array}$} & ptla & $3(23.0)$ \\
\hline & рT2a & $6(46.2)$ \\
\hline & pT2b & $4(30.8)$ \\
\hline & IA & $2(15.4)$ \\
\hline & IB & $2(15.4)$ \\
\hline & $\| \mathrm{A}$ & $3(23.0)$ \\
\hline & $\| B$ & $4(30.8)$ \\
\hline & $\| I I A$ & $2(15.4)$ \\
\hline \multirow{3}{*}{ Lobe distribution } & Left lower lobe & $7(53.8)$ \\
\hline & Right lower lobe & $4(30.8)$ \\
\hline & Right middle lobe & $2(15.4)$ \\
\hline \multirow{2}{*}{ Type of operation } & Lobectomy & $11(84.6)$ \\
\hline & Bilobectomy & $2(15.4)$ \\
\hline \multirow{2}{*}{ Adjuvant therapy } & Adjuvant chemotherapy & $4(30.8)$ \\
\hline & Adjuvant radiotherapy & $1(7.7)$ \\
\hline
\end{tabular}

Data are presented by mean \pm SD or number $(N, \%)$.

DLCO = diffusing capacity of the lung for carbon monoxide; $F E V_{1}=$ forced expiratory volume in $1 \mathrm{~s}$; $\mathrm{FVC}=$ forced vital capacity demanding as IPF is independently associated with poorer overall survival in patients with lung cancer who undergo pulmonary resection..$^{9-11}$ Surgical procedures can lead to an acute exacerbation of IPF or other respiratory complications. ${ }^{12}$ And as reported, pre-existing interstitial lung disease, such as IPF, is a significant risk factor for developing severe radiation pneumonitis after RT.13-16

This study aimed to assess the frequency of IPF in a group of patients who had radical surgical resection for an early-stage NSCLC, to describe morphological and clinical characteristics and report outcomes of this cohort of patients.

\section{Patients and methods}

We conducted an observational cohort retrospective study analyzing 641 consecutive pathological records of patients who underwent lung surgery because of an early-stage NSCLC at University Clinic Golnik from May 2010 to April 2017. Patients with metastatic NSCLC or neuroendocrine carcinoma were excluded from the analysis.

The study was conducted in accordance with the ethical standards laid down in an appropriate version of the 1964 Declaration of Helsinki. The study was conducted with the understanding and the consent of the subjects. Prior to intervention for the acquisition of bioptic material, patients have signed an informed consent for intervention and that their data can be used for scientific purposes.

All pathological records with an early-stage NSCLC and interstitial fibrosis with UIP pattern were selected for more detailed analysis. CT scans and pathological specimens for this group of patients were reviewed again by a thoracic radiologist and pathologist, independently. The UIP/IPF patient cohort included patients who were given a diagnosis of definite UIP/IPF, probable UIP/IPF, or possible UIP/IPF in according with the American Thoracic Society, European Respiratory Society, Japanese Respiratory society, and Latin American Thoracic association (ATS/ESR/JRS/ALAT) 2011 statement. ${ }^{6}$ All other patients were excluded from further analysis.

The clinical data of patients with coexisting early-stage NSCLC and UIP/IPF were thoroughly reviewed. Demographic data (age, gender, smoking status), pulmonary function test results (forced expiratory volume in $1 \mathrm{~s}\left(\mathrm{FEV}_{1}\right)$, forced vital capacity (FVC), diffusing capacity of the lung for carbon monoxide (DLCO)), as well as data on postoperative morbidity, mortality and adjuvant therapy 
TABLE 2. Baseline clinical, radiological and pathological characteristics of the two patients who died in two months time after radical treatment of early-stage non-small cell lung cancer due to acute exacerbation of idiopathic pulmonary fibrosis (IPF)

\begin{tabular}{lll}
\hline Characteristics & Patient No. 1 & Patient No. 2 \\
\hline Gender & Male & Male \\
Age, years & 73 & 81 \\
Smoking status & Former-smoker & Former-smoker \\
FEV 1 FVC before operation & $72 \%$ & $70 \%$ \\
FEV before operation & $2800 \mathrm{ml}(93 \%)$ & $3110 \mathrm{ml}$ (121\%) \\
FVC before operation & $3900 \mathrm{ml}(94 \%)$ & $4570 \mathrm{ml}(122 \%)$ \\
DLCO before operation & $57 \%$ & $47 \%$ \\
Histological subtype & Squamous cell carcinoma & Squamous cell carcinoma \\
Pathologic TNM stage (8th edition) & IIIA & IA \\
Lobe distribution & Left lower lobe & Left lower lobe \\
Type of operation & Lobectomy & Lobectomy \\
Adjuvant therapy & Chemotherapy and radiotherapy & No \\
Time from the end of radical treatment till death & 54 days after finishing radiotherapy & 14 days after surgical resection \\
\hline
\end{tabular}

$\mathrm{DLCO}=$ diffusing capacity of the lung for carbon monoxide; $\mathrm{FEV}_{1}=$ forced expiratory volume in $1 \mathrm{~s}$; FVC = forced vital capacity

(chemotherapy or RT) for lung cancer or further therapy for UIP/IPF were collected from our hospital data base. Most of the patients were followedup in other institutions, therefore data on pulmonary function tests after radical treatment are missing and not presented in this paper.

The period of data collection was from May 2010 to April 2018.

\section{Results}

Out of 641 patients with an early-stage NSCLC only $13(2.0 \%)$ had histologically and radiologically proven coexisting UIP/IPF.

In this group of patients, $11 / 13(84.6 \%)$ were men, $11 / 13(84.6 \%)$ were current or former smokers. Mean age at time of diagnosis was 73.3 years. Pulmonary function test results before resection $\left(\mathrm{FEV}_{1}, \mathrm{FVC}, \mathrm{DLCO}\right)$ are described in Table 1.

Squamous cell carcinoma was diagnosed in $7 / 13(53.8 \%)$ patients and adenocarcinoma in 5/13 $(38.5 \%)$ patients. Pathological stage I was present in $4 / 13(30.8 \%)$ patients, stage II in 7/13 (53.8\%) patients and stage III in $2 / 13(15.4 \%)$ patients. In $11 / 13$ $(84.6 \%)$ of patients the tumor was located in the lower lobes. Lobectomy was performed in 11/13 $(84.6 \%)$ of patients, other $2 / 13(15.4 \%)$ patients had bilobectomy. After radical surgery, 4/13 (30.8\%) patients received adjuvant chemotherapy and $1 / 13$

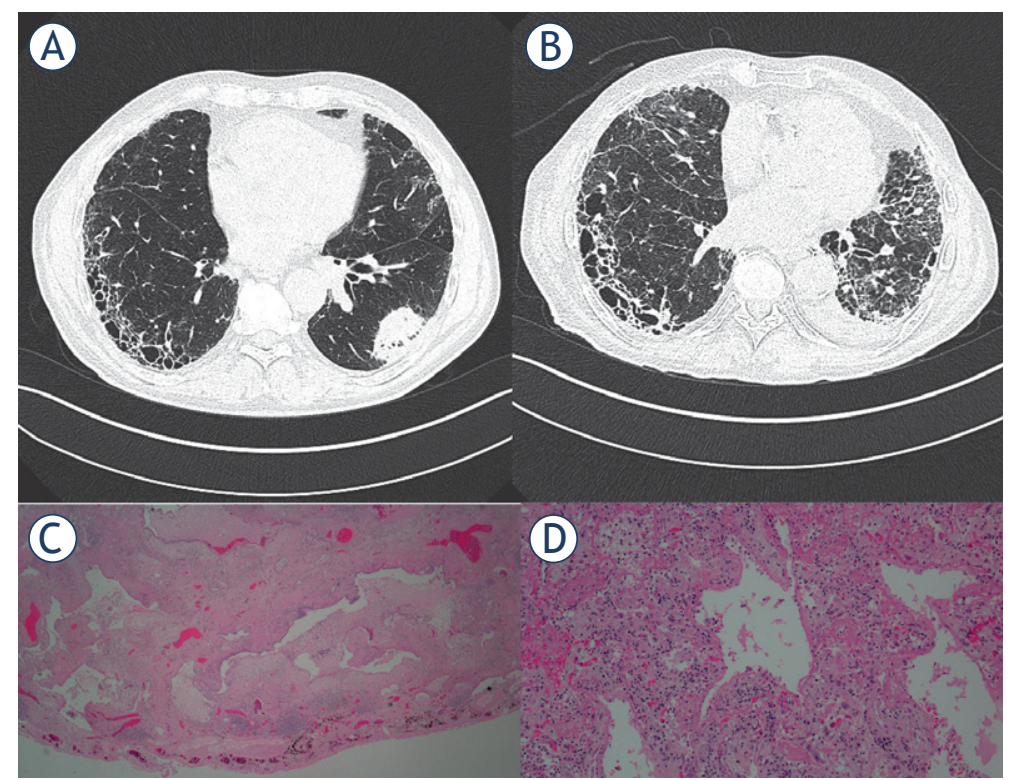

FIGURE 1. Radiological images and images of pathological specimens for Patient No.1. (A) CT scan before lung resection demonstrating honeycomb cystic changes in the subpleural regions of the lung characteristic for typical UIP pattern and tumor in left lower lobe. (B) CT scan after (radical treatment) lobectomy showing extensive bilateral ground glass opacities which are consistant with acute exacerbation of UIP and progressive reticular fibrotic changes. (C) Surgical specimen showing subpleural honeycombing with fibroblastic focus. (D) Autopsy specimen showing acute lung injury with hyaline membranes.

(7.7\%) patient received adjuvant radiotherapy because of $\mathrm{pN} 2$ stage. 


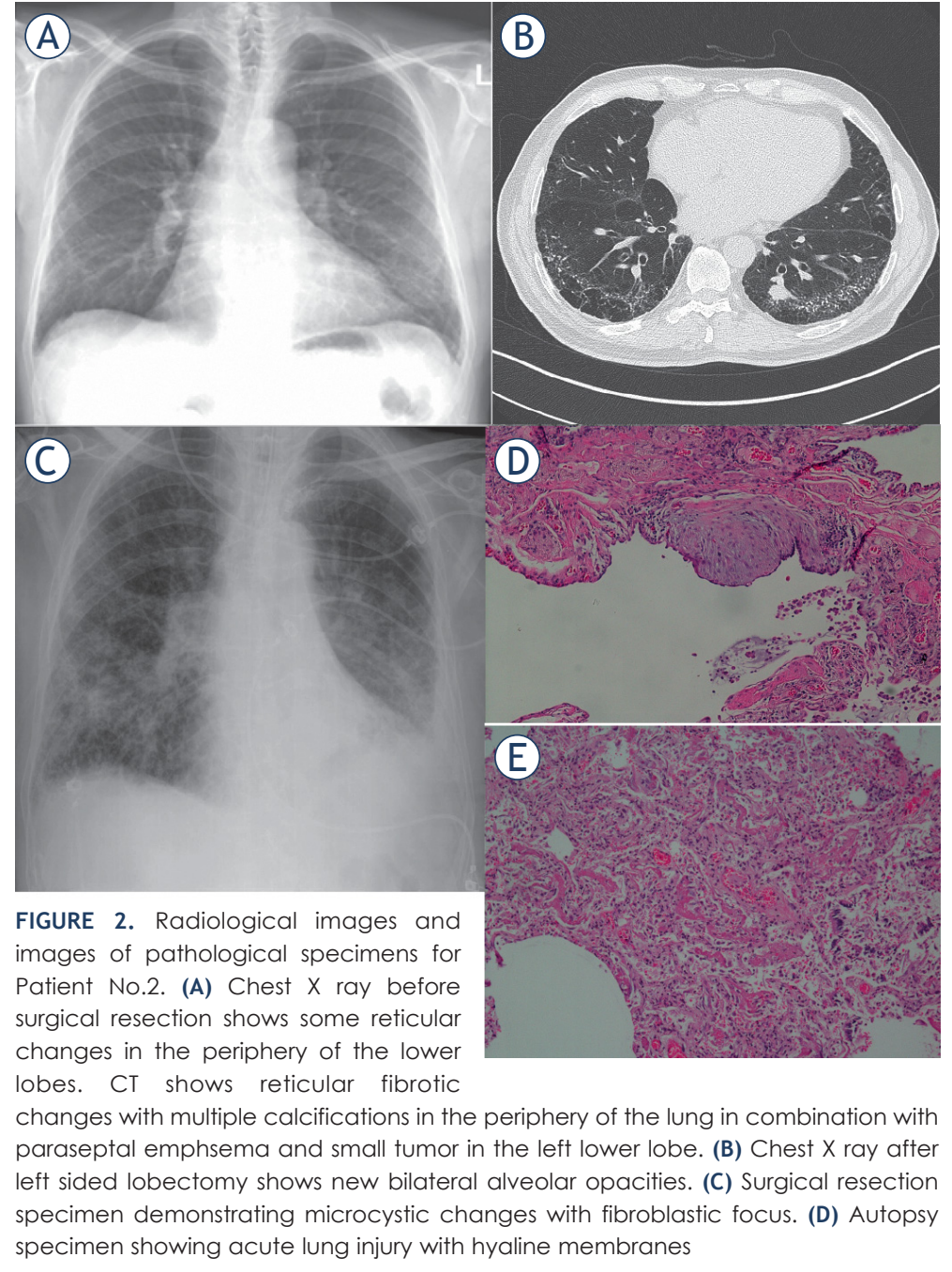

Baseline clinical, pathological and radiological characteristics of patients are shown in Table 1.

There were $2 / 13$ pathologically confirmed fatal cases $(15.4 \%)$ due to acute exacerbation of IPF (aeIPF). Characteristics of these two patients are presented in Table 2. Autopsy was performed in both cases to determine the cause of death. Patient No. 1 started to clinically deteriorate with respiratory insufficiency and dry cough four weeks after finishing postoperative adjuvant RT, and died 54 days after finishing it. In patient No. 2 acute respiratory deterioration developed on the second postoperative day, leading to death on day 14 after surgery. Both patients were treated with high dose corticosteroids and oxygen. Figure 1 and 2 are showing radiological images and images of pathological specimens for these two patients.

Another patient died in the first month after surgery, data on the cause of death is missing.
Three patients (23.1\%) had documented UIP/IPF progression during follow-up in the period of the data collection. They were all regularly seen by an interstitial lung diseases specialist and received pharmacologic therapy for UIP/IPF, one patient was treated with corticosteroid monotherapy and two were treated with pirfenidone.

Out of 13 patients only one (7.7\%) had a lung cancer relapse during data collection period and died because of this.

\section{Discussion}

Based on our one-center retrospective analysis, only $13 / 641(2.0 \%)$ of patents with early NSCLC had concomitant IUP/IPF. To our knowledge, this is the lowest percentage to be reported till now, as earlier reports found incidence from 2.4-24.3\%. ${ }^{17-19}$ The reason is probably the use of more strict inclusion criteria in our study. We followed both histological and radiological criteria for UIP/IPF and not only histological analysis. Another reason could be that surgical resection was performed in fewer patients compared to other studies due to more strict criteria for surgery. Anyhow, looking closely only at the characteristics of the two patients that died because of aeUIP, our criteria for surgery were not strict. They were elderly and had quiet low DLCO. Due to a small number of patients it is difficult to make a general conclusion.

Tumors were mostly small, located in the lower lobes, with squamous histology being more frequent. We noticed male predominance and highly positive smoking status. All this is in accordance with earlier reports. ${ }^{19,20}$

Two patients $(15.4 \%)$ had an aeIPF with fatal outcome after radical therapy, one after surgery and one after postoperative radiotherapy. It is known, that patients with IPF are at very high risk for adverse pulmonary events after lung resection surgery and that the greater the extent of lung resection, the higher incidence of not only aeIPF, but also pneumonia, prolonged air leakage, bronchopleural fistula, empyema in the postoperative period. ${ }^{21,22}$ There are now reports that limited resections are acceptable if the resection can be achieved with an adequate margin. ${ }^{23} \mathrm{New}$ treatment options such as SBRT, which deliver highly conformal, high doses of radiotherapy to clinical target and spare the surrounding tissue, were tested as an alternative to surgery. Yet, also after SBRT there are cases of severe radiation pneumonitis published in the literature. ${ }^{20}$ Regarding which is the standard of 
care in these mostly elderly patients still remains unresolved. There is a great need to conduct a randomized controlled trial to compare survival and quality of life after surgical treatment, either radical or limited resection, versus nonsurgical treatment and to determine predictive factors that would help to guide the decision. Clinical registries could also prospectively collect data on this group of patients, helping to gain more real-life data.

Until we get more of this hard data on which oncological-surgical treatment is most appropriate, it is worthwhile to discuss such clinical cases in an individual manner at a multidisciplinary tumor board, with an interstitial lung disease specialist being part of it. At the same time, these patients should be followed by interstitial lung diseases specialist after completing radical treatment for lung cancer, receiving the appropriate therapy for IPF, if needed. Intensive surveillance is a must in this group of patients.

Our retrospective observational study has many limitations. Our major limitation is a retrospective design of the study. This limited the amount of data available for analysis. As stated before, there are missing data on lung function tests after radical treatment, which would enrich our analysis. Another limitation is that our patients had only performed a CT scan preoperatively, not a highresolution $\mathrm{CT}$, which is an essential component of the diagnostic pathway in IPF. ${ }^{24}$

\section{Conclusions}

The decision on optimal treatment of patients with early-stage NSCLC with concurrent IPF should be made in a multidisciplinary team with close cooperation with specialist for interstitial lung diseases. Not depending on the type of radical treatment, patients with IPF should be then regularly followed by specialist for interstitial lung diseases.

\section{References}

1. Cancer in Slovenia 2015. Ljubljana: Institute of Oncology Ljubljana, Epidemiology and Cancer Registry, Cancer Registry of Republic of Slovenia, 2018.

2. Cufer T, Kosnik M. [Clinical Registry of Lung Cancer Patients]. [Slovenian]. Onkologija 2013; 1: 8-10.

3. Postmus PE, Kerr KM, Oudkerk M, Waller DA, Vansteenkiste J, Escriu C, et al. Early and locally advanced non-small-cell lung cancer (NSCLC): ESMO Clinical Practice Guidelines for diagnosis, treatment and follow-up. Ann Oncol 2017; 28(Suppl 4): 1-21. doi: 10.1093/annonc/mdx222

4. Lindberg K, Nyman J, Kallskog VR, Hoyer M, Lund A, Lax I, et al. Long-term results of a prospective phase II trial of medically inoperable stage I NSCLC treated with SBRT - the Nordic experience. Acta Oncol 2015; 54: 1096-104. doi: 10.3109/0284186X.2015.1020966
5. Verstegen NE, Lagerwaard FJ, Hashemi SM, Dahele M, Slotman BJ, Senan S. Patterns of disease recurrence after SABR for early stage non-small-cell lung cancer: optimizing follow-up schedules salvage therapy. J Thorac Oncol 2015; 10: 1195-200. doi: 10.1097/JTO.0000000000000576

6. Raghu G, Collard HR, Egan JJ, Martinez FJ, Behr J, Brown KK, et al. An official ATS/ERS/JRS/ALAT statement: idiopathic pulmonary fibrosis: evidencebased guidelines for diagnosis and management. Am J Respir Crit Care Med 2011; 183: 788-824. doi: 10.1164/rccm.2009-040GL

7. Rudd RM, Prescott RJ, Chalmers JC, Johnston IDA; Fibrosing Alveolitis Subcommittee of the Research Committee of the British Thoracic Society. British Thoracic Society Study on cryptogenic fibrosing alveolitis: response to treatment and survival. Thorax 2007; 62: 62-66. doi: 10.1136/ thx.2005.045591

8. Raghu G, Amatto VC, Behr J, Stowasser S. Comorbidities in idiopathic pulmonary fibrosis patients: a systematic literature review. Eur Respir J 2015; 46: 1113-30. doi: 10.1183/13993003.02316-2014

9. Omori T, Tajiri M, Baba T, Ogura T, Iwasawa T, Okudela K, et al. Pulmonary resection for lung cancer in patients with idiopathic interstitial pneumonia. Ann Thorac Surg 2015; 100: 954-60. doi: 10.1016/j.athoracsur.2015.03.094

10. Saito Y, Kawai Y, Takahashi N, Ikeya T, Murai K, Kawabata Y, et al. Survival after surgery for pathologic stage IA non-small-cell lung cancer associated with idiopathic pulmonary fibrosis. Ann Thorac Surg 2011; 92: 1812-7. doi: 10.1016/j.athoracsur.2011.06.055

11. Lee T, Park JY, Lee HY, Cho YJ, Yoon HI, Lee JH, et al. Lung cancer in patients with idiopathic pulmonary fibrosis: clinical characteristics and impact on survival. Respir Med 2014; 108: 1549-55. doi: 10.1016/j.rmed.2014.07.020

12. Ghatol A, Ruhl AP, Danoff SK. Exacerbations in idiopathic pulmonary fibrosis triggered by pulmonary and nonpulmonary surgery: a case series and comprehensive review of the literature. Lung 2012; 190: 373-80. doi: 10.1007/ s00408-012-9389-5

13. Ueki N, Matsuo $Y$, Togashi $Y$, Kubo T, Shibuya K, lizuka $Y$, et al. Impact of pretreatment interstitial lung disease on radiation pneumonitis and survival after stereotactic body radiation therapy for lung cancer. J Thorac Oncol 2015: 10: 116-25. doi: 10.1097/JTO.0000000000000359

14. Isobe K, Hata Y, Sakamoto S, Takai y, Shibuya K, Homma S. Clinical characteristics of acute respiratory deterioration in pulmonary fibrosis associated with lung cancer following anti-cancer therapy. Respirology 2010; 15: 88-92. doi: 10.1111/j.1440-1843.2009.01666.x

15. Yamaguchi S, Ohguri T, Ide S, Aoki T, Imada H, Yahara K, et al. Stereotactic body radiotherapy for lung tumors in patients with subclinical interstitial lung disease: the potential risk of extensive radiation pneumonitis. Lung Cancer 2013; 82: 260-5. doi: 10.1016/j.lungcan.2013.08.024

16. Takeda A, Sanuki N, Enomoto T, Kunieda E. Subclinical interstitial lung disease: is it a risk factor for fatal radiation pneumonitis following stereotactic body radiotherapy? Lung Cancer 2014; 83: 112. doi: 10.1016/j. lungcan.2013.10.009

17. Goto T, Maeshima A, Oyamada Y, Kato R. Idiopathic pulmonary fibrosis as a prognostic factor in non-small cell lung cáncer. Int J Clin Oncol 2014; 19: 266-73. doi: 10.1007/s10147-013-0566-1

18. Kawasaki H, Nagai K, Yoshida J, Nishimura M, Nishiwaki Y. Postoperative morbidity, mortality, and survival in lung cancer associated with idiopathic pulmonary fibrosis. J Surg Oncol 2002; 81: 33-7. doi: 10.1002/jso.10145

19. Naccche J, Gibiot Q, Monnet I, Antoine M, Wislez M, Chouaid C, et al. Lung cancer and interstitial lung disease: a literature review. J Thorac Dis 2018; 10: 3829-44. doi: 10.21037/jtd.2018.05.75

20. Khan KA, Kennedy MP, Moore E, Crush L, Prendeville S, Maher MM, et al. Radiological characteristics, histological features and clinical outcomes of lung cancer patients with coexistent idiopathic pulmonary fibrosis. Lung 2015; 193: 71-7. doi: 10.1007/s00408-014-9664-8

21. Watanabe A, Miyajima M, Mishina T, Nakazawa J, Harada R, Kawaharada N et al. Surgical treatment for primary lung cancer combined with idiopathic pulmonary fibrosis. Gen Thorac Cardiovasc Surg 2013; 61: 254-61. doi: 10.1007/s11748-012-0180-6

22. Watanabe A, Higami T, Ohori S, Koyanagi T, Nakashima S. Mawatari T. Is lung cancer resection indicated in patients with idiopathic pulmonary fibrosis? J Thorac Cardiovasc Surg 2008; 136: 1357-63. doi: 10.1016/j. jtcvs.2008.07.016

23. Fujimoto T, Okazaki T, Matsukura T, Hanawana T, Yamashita N, Nishimura $\mathrm{K}$, et al. Operation for lung cancer in patients with idiopathic pulmonary fibrosis: surgical contraindication? Ann Thorac Surgery 2003; 76: 1674-9. doi: 10.1016/S0003-4975(03)00966-4

24. Martinez FJ, Chisholm A, Collard HR, Flaherty KR, Myers J, Raghu G, et al. The diagnosis of idiopathic pulmonary fibrosis: current and future approaches. Lancet Respir Med 2017; 5: 61-71. doi: 10.1016/S2213-2600(16)30325-3 